\title{
A Ruelle Operator for continuous time Markov Chains
}

\author{
Alexandre Baraviera ${ }^{*}$ Artur O. Lopes ${ }^{\dagger}$ Ruy Exel ${ }^{\ddagger}$ \\ * † Instituto de Matemática, UFRGS, Porto Alegre 91501-970, Brasil \\ ‡ Departamento de Matemática, UFSC, Florianopolis 88040-900, Brasil
}

Resumo. We consider a generalization of the Ruelle theorem for the case of continuous time problems. We present a result which we believe is important for future use in problems in Mathematical Physics related to $C^{*}$-Algebras.

We consider a finite state set $S$ and a stationary continuous time Markov Chain $X_{t}, t \geq 0$, taking values on $S$. We denote by $\Omega$ the set of paths $w$ taking values on $S$ (the elements $w$ are locally constant with left and right limits and are also right continuous on $t$ ). We consider an infinitesimal generator $L$ and a stationary vector $p_{0}$. We denote by $P$ the associated probability on $(\Omega, \mathcal{B})$. All functions $f$ we consider bellow are in the set $\mathcal{L}^{\infty}(P)$.

From the probability $P$ we define a Ruelle operator $\mathcal{L}^{t}, t \geq 0$, acting on functions $f: \Omega \rightarrow \mathbb{R}$ of $\mathcal{L}^{\infty}(P)$. Given $V: \Omega \rightarrow \mathbb{R}$, such that is constant in sets of the form $\left\{X_{0}=c\right\}$, we define a modified Ruelle operator $\tilde{\mathcal{L}}_{V}^{t}, t \geq 0$, in the following way: there exist a certain $f_{V}$ such that for each $t$ we consider the operator acting on $g$ given by

$$
\tilde{\mathcal{L}}_{V}^{t}(g)(w)=\left[\frac{1}{f_{V}} \mathcal{L}^{t}\left(e^{\int_{0}^{t}\left(V \circ \Theta_{s}\right)(.) d s} g f_{V}\right)\right](w)
$$

We are able to show the existence of an eigenfunction $u$ and an eigen-probability $\nu_{V}$ on $\Omega$ associated to $\tilde{\mathcal{L}}_{V}^{t}, t \geq 0$.

We also show the following property for the probability $\nu_{V}$ : for any integrable $g \in \mathcal{L}^{\infty}(P)$ and any real and positive $t$

$$
\int e^{-\int_{0}^{t}\left(V \circ \Theta_{s}\right)(.) d s}\left[\left(\tilde{\mathcal{L}}_{V}^{t}(g)\right) \circ \theta_{t}\right] d \nu_{V}=\int g d \nu_{V}
$$

This equation generalize, for the continuous time Markov Chain, a similar one for discrete time systems (and which is quite important for understanding the KMS states of certain $C^{*}$-algebras).

\footnotetext{
* Beneficiary of grant given by CAPES - PROCAD.

$\dagger$ Partially supported by CNPq, Instituto do Milenio, PRONEX - Sistemas Dinâmicos, INCT em Matemática - CNPq, Beneficiary of grant given by CAPES - PROCAD.

‡ Partially supported by CNPq, Instituto do Milenio, PRONEX - Sistemas Dinâmicos.
} 


\section{Introduction}

We want to extend the concept of Ruelle operator to continuous time Markov Chains. In order to do that we need a probability a priori on paths. This fact is not explicit in the discrete time case (thermodynamic formalism) but it is necessary here.

We consider a continuous time stochastic process: the sample paths are functions of the positive real line $\mathbb{R}_{+}=\{t \in \mathbb{R}: t \geq 0\}$ taking values in a finite set $S$ with $n$ elements, that we denote by $S=\{1,2, \ldots, n\}$. Now, consider a $n$ by $n$ real matrix $L$ such that:

1) $0<-L_{i i}$, for all $i \in S$,

2) $L_{i j} \geq 0$, for all $i \neq j, i \in S$,

3) $\sum_{i=1}^{n} L_{i j}=0$ for all fixed $j \in S$.

We point out that, by convention, we are considering column stochastic matrices and not line stochastic matrices (see [N] section 2 and 3 for general references).

We denote by $P^{t}=e^{t L}$ the semigroup generated by $L$. The left action of the semigroup can be identified with an action over functions from $S$ to $\mathbb{R}$ (vectors in $\mathbb{R}^{n}$ ) and the right action can be identified with action on measures on $S$ (also vectors in $\mathbb{R}^{n}$ ).

The matrix $e^{t L}$ is column stochastic, since from the assumptions on $L$ it follows that

$$
(1, \ldots, 1) e^{t L}=(1, \ldots, 1)\left(I+t L+\frac{1}{2} t^{2} L^{2}+\cdots\right)=(1, \ldots, 1) .
$$

It is well known that there exist a vector of probability $p_{0}=\left(p_{0}^{1}, p_{0}^{2}, \ldots\right.$, $\left.p_{0}^{n}\right) \in \mathbb{R}^{n}$ such that $e^{t L}\left(p_{0}\right)=P^{t} p_{0}=p^{0}$ for all $t>0$. The vector $p_{0}$ is a right eigenvector of $e^{t L}$. All entries $p_{0}^{i}$ are strictly positive, as a consequence of hypothesis 1 .

Now, let's consider the space $\tilde{\Omega}=\{1,2, \ldots, n\}^{\mathbb{R}_{+}}$of all functions from $\mathbb{R}_{+}$ to $S$. In principle this seems to be enough for our purposes, but technical details in the construction of probability measures on such a space force us to use a restriction: we consider the space $\Omega \subset \tilde{\Omega}$ as the set of rightcontinuous functions from $\mathbb{R}_{+}$to $S$, which also have left limits in every $t>0$. These functions are constant in intervals (closed in the left and open in the right). In this set we consider the sigma algebra $\mathcal{B}$ generated by the cylinders of the form

$$
\left\{w_{0}=a_{0}, w_{t_{1}}=a_{1}, w_{t_{2}}=a_{2}, \ldots, w_{t_{r}}=a_{r}\right\},
$$


where $t_{i} \in \mathbb{R}_{+}, r \in \mathbb{Z}^{+}, a_{i} \in S$ and $0<t_{1}<t_{2}<\ldots<t_{r}$. It is possible to endow $\Omega$ with a metric, the Skorohod-Stone metric $d$, which makes $\Omega$ complete and separable ([EK] section 3.5), but the space is not compact.

Now we can introduce a continuous time version of the shift map as follows: we define for each fixed $s \in \mathbb{R}_{+}$the $\mathcal{B}$-measurable transformation $\Theta_{s}: \Omega \rightarrow \Omega$ given by $\Theta_{s}\left(w_{t}\right)=w_{t+s}$ (we remark that $\Theta_{s}$ is also a continuous transformation with respect to the Skorohod-Stone metric $d$ ).

For $L$ and $p_{0}$ fixed as above we denote by $P$ the probability on the sigma-algebra $\mathcal{B}$ defined for cylinders by

$$
P\left(\left\{w_{0}=a_{0}, w_{t_{1}}=a_{1}, \ldots, w_{t_{r}}=a_{r}\right\}\right)=P_{a_{r} a_{r-1}}^{t_{r}-t_{r-1}} \ldots P_{a_{2} a_{1}}^{t_{2}-t_{1}} P_{a_{1} a_{0}}^{t_{1}} p_{0}^{a_{0}} .
$$

For further details of the construction of this measure we refer the reader to $[\mathrm{B}]$.

The probability $P$ on $(\Omega, \mathcal{B})$ is stationary in the sense that for any integrable function $f$, and, any $s \geq 0$

$$
\int f(w) d P(w)=\int\left(f \circ \Theta_{s}\right) d P(w) .
$$

From now on the Stationary Process defined by $P$ is denoted by $X_{t}$ and all functions $f$ we consider are in the set $\mathcal{L}^{\infty}(P)$.

There exist a version of $P$ such that for a set of full measure we have that all sample elements $w$ are locally constant on $t$, with left and right limits, and $w$ is right continuous on $t$. We consider from now on such probability $P$ acting on this space (see $[\mathrm{EK}]$ chapter 3 ).

From $P$ we are able to define a continuous time Ruelle operator $\mathcal{L}^{t}, t>0$, acting on functions $f: \Omega \rightarrow \mathbb{R}$ of $\mathcal{L}^{\infty}(P)$. It's also possible to introduce the endomorphism $\alpha_{t}: \mathcal{L}^{\infty}(P) \rightarrow \mathcal{L}^{\infty}(P)$ defined as

$$
\alpha_{t}(\varphi)=\varphi \circ \Theta_{t}, \quad \forall \varphi \in \mathcal{L}^{\infty}(P) .
$$

We relate in the next section the conditional expectation with respect to the $\sigma$-algebras $\mathcal{F}_{t}^{+}$with the operators $\mathcal{L}^{t}$ and $\alpha_{t}$, as follows:

$$
\left[\mathcal{L}^{t}(f)\right]\left(\Theta_{t}\right)=E\left(f \mid \mathcal{F}_{t}^{+}\right) .
$$

Given $V: \Omega \rightarrow \mathbb{R}$, such that it is constant in sets of the form $\left\{X_{0}=c\right\}$ (i.e., $V$ depends only on the value of $w(0)$ ), we consider a Ruelle operator family $\tilde{\mathcal{L}}_{V}^{t}$, for all $t>0$, given by

$$
\tilde{\mathcal{L}}_{V}^{t}(g)(w)=\left[\frac{1}{f_{V}} \mathcal{L}^{t}\left(e^{\int_{0}^{t}\left(V \circ \Theta_{s}\right)(.) d s} g f_{V}\right)\right](w),
$$

for any given $g$, where $f_{V}$ is a fixed function. 
We are able to show the existence of an eigen-probability $\nu_{V}$ on $\Omega$, for the family $\tilde{\mathcal{L}}_{V}^{t}$, for all $t>0$, such that satisfies:

Theorem A. For any integrable $g \in \mathcal{L}^{\infty}(P)$ and any positive $t$

$$
\int\left[\frac{1}{e^{\int_{0}^{t}\left(V \circ \Theta_{s}\right)(.) d s} f_{V}}\right]\left[\mathcal{L}^{t}\left(\left[e^{\int_{0}^{t}\left(V \circ \Theta_{s}\right)(.) d s} f_{V}\right] g\right) \circ \Theta_{t}\right] d \nu_{V}=\int g d \nu_{V} .
$$

The above functional equation is a natural generalization (for continuous time) of the similar one presented in Theorem 7.4 in [EL1] and proposition 2.1 in [EL2] .

In [EL1] and [EL2] the important probability in the Bernoulli space is an eigen-probability $\nu$ for the Ruelle operator associated to a certain potential $V=\log H:\{1,2, . ., d\}^{\mathbb{N}} \rightarrow \mathbb{R}$. This probability $\nu$ satisfies: for any $m \in \mathbb{N}$, $g \in C(X)$,

where

$$
\int \lambda_{m} E_{m}\left(\lambda_{m}^{-1} g\right) d \nu=\int g d \nu
$$

$$
\begin{gathered}
\lambda_{m}(x)=\frac{H^{\beta[m]}}{\Lambda^{[m]}}(x)=\frac{H^{\beta}(x) H^{\beta}(\sigma(x)) \ldots H^{\beta}\left(\sigma^{n-1}(x)\right)}{\Lambda^{[m]}(x)}= \\
\frac{e^{\log \left(H^{\beta}(x)\right)+\log \left(H^{\beta}(\sigma(x))\right)+\ldots+\log \left(H^{\beta}\right)\left(\sigma^{n-1}(x)\right)}}{\Lambda^{[m]}(x)},
\end{gathered}
$$

and $\sigma$ is the shift on the Bernoulli space $\{1,2, . ., d\}^{\mathbb{N}}$. Here $E_{m}(f)=$ $E\left(f \mid \sigma^{-m}(\mathcal{B})\right)$ denotes the expected projection (with respect to a initial probability $P$ on the Bernoulii space) on the sigma-algebra $\sigma^{-m}(\mathcal{B})$, where $\mathcal{B}$ is the Borel sigma-algebra and $\Lambda^{[m]}$ is associated to the Jones index.

We refer the reader to [CL] for a Thermodynamic view of $C^{*}$-Algebras which include concepts like pressure, entropy, etc..

We believe it will be important in the analysis of certain $C^{*}$ algebras associated to continuous time dynamical systems a characterization of KMS states by means of the above theorem. We point out, however, that we are able to show this theorem for a certain $\rho_{V}$ just for a quite simple function $V$ as above. In a forthcoming paper we will consider more general potentials $V$.

One could consider a continuous time version of the $C^{*}$-algebra considered in [EL1]. We just give an idea of what we are talking about. Given the above defined $P$ for each $t>0$, denote by $s_{t}: \mathcal{L}^{2}(P) \rightarrow \mathcal{L}^{2}(P)$, the Koopman operator, where for $\eta \in \mathcal{L}^{2}(P)$ we define $\left(s_{t} \eta\right)(x)=\eta\left(\theta_{t}(x)\right)$.

Another important class of linear operators is $M_{f}: \mathcal{L}^{2}(P) \rightarrow \mathcal{L}^{2}(P)$, for a given fixed $f \in C(\Omega)$, and defined by $M_{f}(\eta)(x)=f(x) \eta(x)$, for any $\eta$ in 
$\mathcal{L}^{2}(P)$. We assume that $f$ is such defines an operator on $\mathcal{L}^{2}(P)$ (remember that $\Omega$ is not compact).

In this way we can consider a $C^{*}$-algebra generated by the above defined operators (for all different values of $t>0$ ), then the concept of state, and finally given $V$ and $\beta$ we can ask about KMS states. There are several technical difficulties in the definition of the above $C^{*}$-algebra, etc... Anyway, at least formally, there is a need for finding $\nu$ which is a solution of an equation of the kind we describe here. We need this in order to be able to obtain a characterization of KMS states by means of an eigen-probability for the continuous time Ruelle operator. This setting will be the subject of a future work. This was the motivation for our result.

With the operators $\alpha$ and $\mathcal{L}$ we can rewrite the theorem above as

$$
\rho_{V}\left(G_{T}^{-1} E_{T}\left(G_{T} \varphi\right)\right)=\rho_{V}(\varphi),
$$

for all $\varphi \in \mathcal{L}^{\infty}$ and all $T>0$, where, as usual, $\rho_{V}(\varphi)=\int \varphi d \rho_{V}, E_{T}=\alpha_{T} \mathcal{L}^{T}$ is in fact a projection on a subalgebra of $\mathcal{B}$, and $G_{T}: \Omega \rightarrow \mathbb{R}$ is given by

$$
G_{T}(x)=\exp \left(\int_{0}^{T} V(x(s)) d s\right) .
$$

For the map $V: \Omega \rightarrow \mathbb{R}$, which is constant in cylinders of the form $\left\{w_{0}=i\right\}, i \in\{1,2, \ldots, n\}$, we denote by $V_{i}$ the corresponding value. We also denote by $V$ the diagonal matrix with the $i$-diagonal element equal to $V_{i}$.

Now, consider $P_{V}^{t}=e^{t(L+V)}$. The classical Perron-Frobenius Theorem for such semigroup will be one of the main ingredients of our main proof.

As usual, we denote by $\mathcal{F}_{s}$ the sigma-algebra generated by $X_{s}$. We also denote by $\mathcal{F}_{s}^{+}$the sigma-algebra generated $\sigma\left(\left\{X_{u}, s \leq u\right\}\right)$. Note that a $\mathcal{F}_{s}^{+}$-measurable function $f(w)$ on $\Omega$ does depend of the value $w_{s}$.

We also denote by $I_{A}$ the indicator function of a measurable set $A$ in $\Omega$.

In a forthcoming paper we will analyze the case where the potential $V$ is of a more general type (not just $V: \Omega \rightarrow \mathbb{R}$, such that it's constant in sets of the form $\left\{X_{0}=c\right\}$ )

\section{A continuous time Ruelle Operator}

We present a quite general definition: let $\mathbb{X}$ and $X$ be a separable metric Radon spaces, $\hat{\mu}$ probability on $\mathbb{X}, \pi: \mathbb{X} \rightarrow X$ Borel mensurable and $\mu=\pi_{*} \hat{\mu}$. Then there exists a Borel family of probabilities $\{\hat{\mu}\}_{x \in X}$ on $\mathbb{X}$, uniquely determined $\mu$-a.e, such that,

1) $\hat{\mu}_{x}\left(\mathbb{X} \backslash \pi^{-1}(x)\right)=0$, $\mu$-a.e;

2) $\int g(z) d \hat{\mu}(z)=\int_{X} \int_{\pi^{-1}(x)} g(z) d \hat{\mu}_{x}(z) d \mu(x)$. 
We refer the reader to [AGS] for the proof. Here we will need a more simple version of this general result that can be obtained in an explicit form.

We consider the disintegration of $P$ given by the family of measures, indexed by the elements of $\Omega$ and $t>0$ defined as follows: first, consider a sequence $0=t_{0}<t_{1}<\cdots<t_{j-1}<t \leq t_{j}<\cdots<t_{r}$. Then for $w \in \Omega$ and $t>0$ we have on cylinders:

$$
\begin{gathered}
\mu_{t}^{w}\left(\left[X_{0}=a_{0}, \ldots, X_{t_{r}}=a_{r}\right]\right)= \\
\left\{\begin{array}{cc}
\frac{1}{p_{0}^{w(t)}} P_{w(t) a_{j-1}}^{t-t_{j-1}} \ldots P_{a_{2} a_{1}}^{t_{2}-t_{1}} P_{a_{1} a_{0}}^{t} p_{0}^{a_{0}} & \text { if } a_{j}=w\left(t_{j}\right), \ldots, a_{r}=w\left(t_{r}\right) \\
0 & \text { otherwise. }
\end{array}\right.
\end{gathered}
$$

Proposition 2.1. Under the above conditions on the sequence $0=t_{0}<$ $t_{1}<\cdots<t_{j-1}<t \leq t_{j}<\cdots<t_{r}$, we have that $\mu_{t}^{w}$ is the disintegration of $P$ along the fibers $\Theta_{t}^{-1}($.$) .$

Proof: It is enough to show that for any integrable $f$

$$
\int_{\Omega} f d P=\int_{\Omega} \int_{\Theta_{t}^{-1}(w)} f(x) d \mu_{t}^{w}(x) d P(w) .
$$

For doing that we can assume that $f$ is in fact the indicator of the cylinder $\left[X_{0}=a_{0}, \ldots, X_{t_{r}}=a_{r}\right]$; then the right hand side becomes

$$
\begin{gathered}
\iint f d \mu_{t}^{w}(x) d P(w)= \\
\int I_{\left[w\left(t_{j}\right)=a_{j}, \ldots, w\left(t_{r}\right)=a_{r}\right]} \frac{1}{p_{0}^{w(t)}} P_{w(t) a_{j-1}}^{t-t_{j-1}} \ldots P_{a_{1} a_{0}}^{t_{1}} p_{0}^{a_{0}} d P(w)= \\
\sum_{a=1}^{n} \int I_{\left[w(t)=a, w\left(t_{j}\right)=a_{j}, \ldots, w\left(t_{r}\right)=a_{r}\right]} \frac{1}{p_{0}^{w(t)}} P_{w(t) a_{j-1}}^{t-t_{j-1}} \ldots P_{a_{1} a_{0}}^{t_{1}} p_{0}^{a_{0}} d P(w)= \\
\sum_{a=1}^{n} P_{a_{r} a_{r-1}}^{t_{r}-t_{r-1}} \ldots P_{a_{j} a}^{t_{j}-t} p_{0}^{a} \frac{1}{p_{0}^{a}} P_{a a_{j-1}}^{t-t_{j-1}} \cdots P_{a_{1} a_{0}}^{t_{1}} p_{0}^{a_{0}}=P_{a_{r} a_{r-1}}^{t_{r}-t_{r-1}} \cdots P_{a_{1} a_{0}}^{t_{1}} p_{0}^{a_{0}}= \\
P\left(\left[X_{0}=a_{0}, \ldots, X_{t_{r}}=a_{r}\right]\right)=\int f d P .
\end{gathered}
$$

In the second inequality we use the fact that $P$ is stationary.

The proof for a general $f$ follows from standard arguments. 
Definition 2.2. For $t$ fixed we define the operator $\mathcal{L}^{t}: \mathcal{L}^{\infty}(\Omega, P) \rightarrow$ $\mathcal{L}^{\infty}(\Omega, P)$ as follows:

$$
\mathcal{L}^{t}(\varphi)(x)=\int_{\bar{y} \in \Theta_{t}^{-1}(x)} \varphi(\bar{y}) d \mu_{t}^{x}(\bar{y}) .
$$

Remark 2.3. The definition above can be rewritten as

$$
\mathcal{L}^{t}(\varphi)(x)=\int_{y \in D[0, t)} \varphi(y x) d \mu_{t}^{x}(y x),
$$

where the symbol yx means the concatenation of the path $y$ with the translation of $x$ :

$$
x y(s)=\left\{\begin{array}{cc}
y(s) & \text { if } s \in[0, t) \\
x(s-t) & \text { if } s \geq t
\end{array}\right.
$$

and, $D[0, t)$ is the set of right-continuous functions from $[0, t)$ to $S$. This follows simply from the fact that, in this notation, $\Theta_{t}^{-1}(x)=\{y x: y \in$ $D[0, t)\}$.

Note that the value $\lim _{s \rightarrow t} y(s)$ do not have to be necessarily equal to $x(0)$.

In order to understand better the definitions above we apply the operator to some simple functions. For example, we can see the effect of $\mathcal{L}^{t}$ on some indicator function of a given cylinder: consider the sequence $0=t_{0}<t_{1}<$ $. .<t_{j-1}<t \leq t_{j}<\ldots<t_{r}$ and then take $f=I_{\left\{X_{0}=a_{0}, X_{t_{1}}=a_{1}, \ldots, X_{t_{r}}=a_{r}\right\}}$. Then, for a path $z \in \Omega$ such that $z_{t_{j}-t}=a_{j}, \ldots, z_{t_{r}-t}=a_{r}$ (the future condition) we have

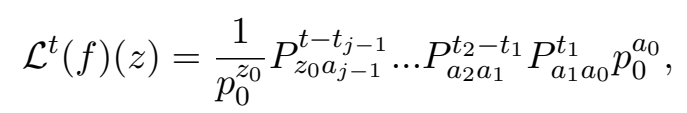

otherwise (i.e., if the path $z$ does not satisfy the condition above) we get $\mathcal{L}^{t}(f)(z)=0$.

Note that if $t_{r}<t$, then $\mathcal{L}^{t}(f)(z)$ depends only on $z_{0}$. For example, if $f=I_{\left\{X_{0}=i_{0}\right\}}$ then

$$
\mathcal{L}^{t}(f)(z)=\int_{y \in D[0, t)} I_{\left\{X_{0}=i_{0}\right\}}(y x) d \mu_{t}^{z}(y x)=\mu_{t}^{z}\left(\left[X_{0}=i_{0}\right]\right)=\frac{1}{p_{0}^{z_{0}}} P_{z_{0}, i_{0}}^{t} p_{0}^{i_{0}} .
$$

In the case $f=I_{\left\{X_{0}=i_{0}, X_{t}=j_{0}\right\}}$, then $\mathcal{L}^{t}(f)(z)=P_{z_{0}, i_{0}}^{t} \frac{p_{0}^{i_{0}}}{p_{0}^{z_{0}}}$, if $z_{0}=j_{0}$, and $\mathcal{L}^{t}(f)(z)=0$ otherwise.

We describe bellow some properties of $\mathcal{L}^{t}$.

Proposition 2.4. $\mathcal{L}^{t}(1)=1$, where 1 is the function that maps every point in $\Omega$ to 1. 
Proof: Indeed,

$$
\begin{gathered}
\mathcal{L}^{t}(1)(x)=\int_{y \in D[0, t)} 1(y x) d \mu_{t}^{x}(y x)=\int d \mu_{t}^{x}(y x)=\mu_{t}^{x}\left(\left[X_{t}=x(0)\right]\right)= \\
\sum_{a=1}^{n} \mu_{t}^{x}\left(\left[X_{0}=a, X_{t}=x(0)\right]\right)=\frac{1}{p_{0}^{x(0)}} \sum_{a=1}^{n} P_{x(0) a}^{t} p_{0}^{a}=1 .
\end{gathered}
$$

We can also define the dual of $\mathcal{L}^{t}$, denoted by $\left(\mathcal{L}^{t}\right)^{*}$, acting on the measures. Then we get:

Proposition 2.5. For any positive $t$ we have that $\left(\mathcal{L}^{t}\right)^{*}(P)=(P)$.

Proof: For a fixed $t$ we have that $\left(\mathcal{L}^{t}\right)^{*}(P)=(P)$, because for any $f$ of the form $f=I_{\left\{X_{0}=a_{0}, X_{t_{1}}=a_{1}, \ldots, X_{t_{r}}=a_{r}\right\}}, 0=t_{0}<t_{1}<. .<t_{j-1}<t \leq t_{j}<$ $\ldots<t_{r}$, we get

$$
\begin{aligned}
& \int \mathcal{L}^{t}(f)(z) d P(z)=\sum_{b=1}^{n} \int_{\left\{X_{0}=b\right\}} \mathcal{L}^{t}(f)(z) d P(z)= \\
& \sum_{b=1}^{n} \int I_{\left\{X_{0}=b, X_{t_{j}-t}=a_{j}, \ldots, X_{t_{r}-t}=a_{r}\right\}}(z) d P(z) \frac{1}{p_{0}^{b}} P_{b a_{j-1}}^{t-t_{j-1}} \ldots P_{a_{2} a_{1}}^{t_{2}-t_{1}} P_{a_{1} a_{0}}^{t_{1}} p_{0}^{a_{0}}= \\
& \sum_{b=1}^{n} P\left(\left\{X_{0}=b, X_{t_{j}-t}=a_{j}, \ldots, X_{t_{r}-t}=a_{r}\right\}\right) \frac{1}{p_{0}^{b}} P_{b a_{j-1}}^{t-t_{j-1}} \ldots P_{a_{2} a_{1}}^{t_{2}-t_{1}} P_{a_{1} a_{0}}^{t_{1}} p_{0}^{a_{0}}= \\
& \sum_{b=1}^{n} P_{a_{r} a_{r-1}}^{t_{r}-t_{r-1}} \ldots P_{a_{j+1} a_{j}}^{t_{j+1}-t_{j}} P_{a_{j} b}^{t_{j}-t} p_{0}^{b} \frac{1}{p_{0}^{b}} P_{b a_{j-1}}^{t-t_{j-1}} \ldots P_{a_{2} a_{1}}^{t_{2}-t_{1}} P_{a_{1} a_{0}}^{t_{1}} p_{0}^{a_{0}}= \\
& \int f(w) d P(w)
\end{aligned}
$$

Proposition 2.6. Given $t \in \mathbb{R}_{+}$, and the functions $\varphi, \psi \in \mathcal{L}^{\infty}(P)$, then

$$
\mathcal{L}^{t}\left(\varphi \times\left(\psi \circ \Theta_{t}\right)\right)(z)=\psi(z) \times \mathcal{L}^{t}(\varphi)(z) .
$$

Proof: Using the formula of $\mathcal{L}^{t}$ given by Remark 2.3 we get

$$
\mathcal{L}^{t}\left(\varphi\left(\psi \circ \Theta_{t}\right)\right)(x)=\int_{i \in D[0, t)} \varphi(i x)\left(\psi \circ \Theta_{t}\right)(i x) d \mu_{t}^{x}(i)=
$$




$$
\psi(x) \int_{i \in D[0, t)} \varphi(i x) d \mu_{t}^{x}(i)=\left(\psi \mathcal{L}^{t}(\varphi)\right)(x)=\psi(x) \mathcal{L}^{t}(\varphi)(x),
$$

since $\psi \circ \Theta_{t}(i x)=\psi(x)$, independently of $i$.

We just recall that the last proposition can be restated as

$$
\mathcal{L}^{t}\left(\varphi \alpha_{t}(\psi)\right)=\psi \mathcal{L}^{t}(\varphi) .
$$

Then we get:

Proposition 2.7. $\alpha_{t}$ is the adjoint of $\mathcal{L}^{t}$ on $\mathcal{L}^{2}(P)$.

Proof: From last two propositions

$\int \mathcal{L}^{t}(f) g d P=\int \mathcal{L}^{t}\left(f \times\left(g \circ \Theta_{t}\right)\right) d P=\int f \times\left(g \circ \Theta_{t}\right) d P=\int f \alpha_{t}(g) d P$, as claimed.

We want to obtain conditional expectations in a more explicit form. For a given $f$, recall that the function $Z(w)=E\left(f \mid \mathcal{F}_{t}^{+}\right)$is the $Z$ (almost everywhere defined) $\mathcal{F}_{t}^{+}$-measurable function such that for any $\mathcal{F}_{t}^{+}$-measurable set $B$ we have $\int_{B} Z(w) d P(w)=\int_{B} f(w) d P(w)$.

Proposition 2.8. The conditional expectation is given by

$$
E\left(f \mid \mathcal{F}_{t}^{+}\right)(x)=\int f d \mu_{t}^{x}
$$

Proof: For $t$ fixed, consider a $\mathcal{F}_{t}^{+}$-measurable set $B$. Then we have

$$
\begin{gathered}
\int_{B} \int f d \mu_{t}^{w} d P(w)=\int\left(I_{B}(w) \int f d \mu_{t}^{w}\right) d P(w)= \\
\iint\left(f I_{B}\right) d \mu_{t}^{w} d P(w)=\int f(w) I_{B}(w) d P(w)=\int_{B} f d P .
\end{gathered}
$$

Now we can relate the conditional expectation with respect to the $\sigma$ algebras $\mathcal{F}_{t}^{+}$with the operators $\mathcal{L}^{t}$ and $\alpha_{t}$ as follows:

Proposition 2.9. $\left[\mathcal{L}^{t}(f)\right]\left(\Theta_{t}\right)=E\left(f \mid \mathcal{F}_{t}^{+}\right)$. 
Proof: This follows from the fact that for any $B=\left\{X_{s_{1}}=b_{1}, X_{s_{2}}=\right.$ $\left.b_{2}, \ldots, X_{s_{u}}=b_{u}\right\}$, with $t<s_{1}<\ldots<s_{u}$, we have $I_{B}=I_{A} \circ \Theta_{t}$ for some measurable $A$ and

$$
\begin{gathered}
\int_{B} \mathcal{L}^{t}(f)\left(\Theta_{t}(w)\right) d P(w)=\int I_{B}(w) \mathcal{L}^{t}(f)\left(\Theta_{t}(w)\right) d P(w)= \\
\int\left(I_{A} \circ \Theta_{t}\right)(w) \mathcal{L}^{t}(f)\left(\Theta_{t}(w)\right) d P(w)=\int I_{A}(w) \mathcal{L}^{t}(f)(w) d P(w)= \\
\int I_{A}\left(\Theta_{t}(w)\right) f(w) d P(w)=\int_{B} f(w) d P(w) .
\end{gathered}
$$

\section{The modified operator Ruelle Operator associated to $V$}

We consider $V: \Omega \rightarrow \mathbb{R}$, such that is constant in sets of the form $\left\{X_{0}=\right.$ $c\}$. We are interested in the operator obtained by the perturbation of the operator $\mathcal{L}^{t}$ by $V$.

Definition 3.1. We define $G_{t}: \Omega \rightarrow \mathbb{R}$ as

$$
G_{t}(x)=\exp \left(\int_{0}^{t} V(x(s)) d s\right)
$$

Definition 3.2. We define the $G$-weigthed transfer operator $\mathcal{L}_{V}^{t}: \mathcal{L}^{\infty}(\Omega, P) \rightarrow$ $\mathcal{L}^{\infty}(\Omega, P)$ acting on measurable functions $f$ ( of the form $f=I_{\left\{X_{0}=a_{0}, X_{t_{1}}=a_{1}\right.}$, $\left.\left.\ldots, X_{t_{r}}=a_{r}\right\}\right)$ by

$$
\begin{gathered}
\mathcal{L}_{V}^{t}(f)(w):=\mathcal{L}^{t}\left(G_{t} f\right)= \\
=\mathcal{L}^{t}\left(e^{\int_{0}^{t}\left(V \circ \Theta_{s}\right)(.) d s} f\right)=\sum_{b=1}^{n} \mathcal{L}^{t}\left(e^{\int_{0}^{t}\left(V \circ \Theta_{s}\right)(.) d s} I_{\left\{X_{t}=b\right\}} f\right)(w) .
\end{gathered}
$$

Note that $e^{\int_{0}^{t}\left(V \circ \Theta_{s}\right)(.) d s} I_{\left\{X_{t}=b\right\}}$ does not depend on information for time larger than $t$. In the case $f$ is such that $t_{r} \leq t$ (in the above notation), then $\mathcal{L}_{V}^{t}(f)(w)$ depends only on $w(0)$.

The integration on $s$ above is consider over the open interval $(0, t)$.

We will show next the existence of an eigenfunction and an eigen-measure for such operator $\mathcal{L}_{V}^{t}$. First we need the following:

Theorem (Perron-Frobenius for continuous time). ([S] page 111) Given $L, p_{0}$ and $V$ as above, there exists 
a) a unique positive function $u_{V}: \Omega \rightarrow \mathbb{R}$, constant equal to the value $u_{V}^{i}$ in each cylinder $X_{0}=i, i \in\{1,2, . ., n\}$, (sometimes we will consider $u_{V}$ as a vector in $\left.\mathbb{R}^{n}\right)$.

b) a unique probability vector $\mu_{V}$ in $\mathbb{R}^{n}$ (a probability over over the set $\{1,2, . ., n\}$ such that $\left.\mu_{V}(\{i\})>0, \forall i\right)$, that is,

$$
\sum_{i=1}^{n}\left(u_{V}\right)_{i}\left(\mu_{V}\right)_{i}=1
$$

c) a real positive value $\lambda(V)$, such that for any positive $s$

$$
e^{-s \lambda(V)} u_{V} e^{s(L+V)}=u_{V} .
$$

d) Moreover, for any $v=\left(v_{1}, ., v_{n}\right) \in \mathbb{R}^{n}$

$$
\lim _{t \rightarrow \infty} e^{-t \lambda(V)} v e^{t(L+V)}=\left(\sum_{i=1}^{n} v_{i}\left(\mu_{V}\right)_{i}\right) u_{V},
$$

e) for any positive $s$

$$
e^{-s \lambda(V)} e^{s(L+V)} \mu_{V}=\mu_{V} .
$$

From property e) it follows that

$$
(L+V) \mu_{V}=\lambda(V) \mu_{V},
$$

or

$$
(L+V-\lambda(V) I) \mu_{V}=0 .
$$

From c) it follows that

$$
u_{V}(L+V)=\lambda(V) u_{V}
$$

or

$$
u_{v}(L+V-\lambda(V) I)=0 .
$$

We point out that e) means that for any positive $t$ we have $\left(P_{V}^{t}\right)^{*} \mu_{V}=$ $e^{\lambda(V) t} \mu_{V}$.

Note that when $V=0$, then $\lambda(V)=0, \mu_{V}=p^{0}$ and $u_{V}$ is constant equal to 1.

Now we return to our setting: for each $i_{0}$ and $t$ fixed one can consider the probability $\mu_{i_{0}}^{t}$ defined over the sigma-algebra $\mathcal{F}_{t}^{-}=\sigma\left(\left\{X_{s} \mid s \leq t\right\}\right)$ with support on $\left\{X_{0}=i_{0}\right\}$ such that for cylinder sets with $0<t_{1}<\ldots<t_{r} \leq t$ $\mu_{i_{0}}^{t}\left(\left\{X_{0}=i_{0}, X_{t_{1}}=a_{1}, \ldots, X_{t_{r-1}}=a_{r-1}, X_{t}=j_{0}\right\}\right)=P_{j_{0} a_{r}}^{t-t_{r}} \ldots P_{a_{2} a_{1}}^{t_{2}-t_{1}} P_{a_{1} i_{0}}^{t_{1}}$.

The probability $\mu_{i_{0}}^{t}$ is not stationary. 
We denote by $Q(j, i)_{t}$ the $i, j$ entry of the matrix $e^{t(L+V)}$, that is $\left(e^{t(L+V)}\right)_{j, i}$. It is known $([\mathrm{K}]$ page 52 or $[\mathrm{S}]$ Lemma 5.15$)$ that

$$
\begin{gathered}
Q\left(j_{0}, i_{0}\right)_{t}=E_{\left\{X_{0}=i_{0}\right\}}\left\{e^{\int_{0}^{t}\left(V \circ \Theta_{s}\right)(w) d s} ; X(t)=j_{0}\right\}= \\
\int I_{\left\{X_{t}=j_{0}\right\}} e^{\int_{0}^{t}\left(V \circ \Theta_{s}\right)(w) d s} d \mu_{i_{0}}^{t}(w) .
\end{gathered}
$$

For example,

$$
\int I_{\left\{X_{t}=j_{0}\right\}} e^{\int_{0}^{t}\left(V \circ \Theta_{s}\right)(w) d s} d P=\sum_{i=1,2, . ., n} Q\left(j_{0}, i\right)_{t} p_{i}^{0} .
$$

In the particular case where $V$ is constant equal 0 , then $p^{0}=\mu_{V}$ and $\lambda(V)=0$.

We denote by $f=f_{V}$, where $f(w)=f(w(0))$, the density of probability $\mu_{V}$ in $S$ with respect to the probability $p^{0}$ in $S$.

Therefore, $\int f d p^{0}=1$.

Proposition 3.3. $f_{V}(w)=\frac{\mu_{V}(w)}{p^{0}(w)}=\frac{\left(\mu_{V}\right)_{w(0)}}{\left(p^{0}\right)_{w(0)}}, f_{V}: \Omega \rightarrow \mathbb{R}$, is an eigenfunction for $\mathcal{L}_{V}^{t}$ with eigenvalue $e^{t \lambda(V)}$

Proof: Note that $\frac{\mu_{V}}{p^{0}}=\sum_{c=1}^{n} \frac{\mu_{V}(c)}{p^{0}(c)} I_{\left\{X_{0}=c\right\}}$.

For a given $w$, denote $w(0)$ by $j_{0}$, then conditioning

$$
\mathcal{L}_{V}^{t}\left(\frac{\mu_{V}}{p^{0}}\right)(w)=\sum_{c=1}^{n} \sum_{b=1}^{n} \mathcal{L}_{V}^{t}\left(\frac{\mu_{V}(c)}{p^{0}(c)} I_{\left\{X_{0}=c\right\}} I_{\left\{X_{t}=b\right\}}\right)(w) .
$$

Consider $c$ fixed, then for $b=j_{0}$ we have

$$
\mathcal{L}_{V}^{t}\left(I_{\left\{X_{0}=c\right\}} I_{\left\{X_{t}=b\right\}}\right)(w)=\frac{Q\left(j_{0}, c\right)_{t} p_{c}^{0}}{p_{j_{0}}^{0}},
$$

and for $b \neq j_{0}$, we have $\mathcal{L}_{V}^{t}\left(I_{\left\{X_{0}=c\right\}} I_{\left\{X_{t}=b\right\}}\right)(w)=0$.

Finally, for any $t>0$

$$
\begin{gathered}
\mathcal{L}_{V}^{t}\left(\frac{\mu_{V}}{p^{0}}\right)(w)=\sum_{c=1}^{n} \frac{\mu_{V}(c)}{p^{0}(c)} Q\left(j_{0}, c\right)_{t} \frac{p_{c}^{0}}{p_{j_{0}}^{0}}= \\
\sum_{c=1}^{n} \frac{\mu_{V}(c)}{p^{0}(c)}\left(e^{t(L+V)}\right)_{j_{0}, c} \frac{p_{c}^{0}}{p_{j_{0}}^{0}}=e^{t \lambda(V)} \frac{\left(\mu_{V}\right)_{j_{0}}}{p_{j_{0}}^{0}}=e^{t \lambda(V)}\left(\frac{\mu_{V}}{p^{0}}\right)(w),
\end{gathered}
$$

because $e^{t(L+V)}\left(\mu_{V}\right)=e^{t \lambda(V)}\left(\mu_{V}\right)$. 
Therefore, for any $t>0$ the function $f_{V}=\frac{\mu_{V}}{p^{0}}$ (that depends only on $w(0))$ is an eigenfunction for the operator $\mathcal{L}_{V}^{t}$ associated to the eigenvector $e^{t \lambda(V)}$.

The above result shows that the eigenfunction for the Ruelle operator associated to the potential $V$ is the Radon-Nykodin derivative for $\mu_{V}$ with respect to $p^{0}$;

Definition 3.4. Consider for each $t$ the operator acting on $g$ given by

$$
\hat{\mathcal{L}}_{V}^{t}(g)(w)=\left[\frac{1}{f_{V}} \mathcal{L}^{t}\left(e^{\left.\int_{0}^{t}(V-\lambda(V)) \circ \Theta_{s}\right)(.) d s} g f_{V}\right)\right](w)
$$

From the above $\hat{\mathcal{L}}_{V}^{t}(1)=1$, for all positive $t$.

We present some examples: note that by conditioning, if $g=I_{\left\{X_{0}=a_{0}\right.}$, $\left.X_{t_{1}}=a_{1}, X_{t_{2}}=a_{2}, X_{t}=a_{3}\right\}$, with $0<t_{1}<t_{2}<t$, then

$$
\begin{gathered}
\hat{\mathcal{L}}_{V}^{t}(g)(w)= \\
\frac{1}{p_{a_{3}}^{0}} \frac{p_{a_{3}}^{0}}{\mu_{V}\left(a_{3}\right)} e_{a_{3} a_{2}}^{\left(t-t_{2}\right)(L+V-\lambda I)} e_{a_{2} a_{1}}^{\left(t_{2}-t_{1}\right)(L+V-\lambda I)} e_{a_{1} a_{0}}^{t_{1}(L+V-\lambda I)} \frac{\mu_{V}\left(a_{0}\right)}{p_{a_{0}}^{0}} p_{a_{0}}^{0}= \\
\frac{1}{\mu_{V}\left(a_{3}\right)} e_{a_{3} a_{2}}^{\left(t-t_{2}\right)(L+V-\lambda I)} e_{a_{2} a_{1}}^{\left(t_{2}-t_{1}\right)(L+V-\lambda I)} e_{a_{1} a_{0}}^{t_{1}(L+V-\lambda I)} \mu_{V}\left(a_{0}\right),
\end{gathered}
$$

for $w$ such that $w_{0}=a_{3}$, and $\hat{\mathcal{L}}_{V}^{t}(g)(w)=0$ otherwise.

Moreover, for $g=I_{\left\{X_{0}=a_{0}, X_{t_{1}}=a_{1}, X_{t_{2}}=a_{2}\right\}}$, with $0<t_{1}<t<t_{2}$, then

$$
\hat{\mathcal{L}}_{V}^{t}(g)(w)=\frac{1}{\mu_{V}(a)} e_{a a_{1}}^{\left(t-t_{1}\right)(L+V-\lambda I)} e_{a_{1} a_{0}}^{t_{1}(L+V-\lambda I)} \mu_{V}\left(a_{0}\right),
$$

for $w$ such that $w_{0}=a, w_{t_{2}-t}=a_{2}$, and $\hat{\mathcal{L}}_{V}^{t}(g)(w)=0$ otherwise.

Consider now the dual operator $\left(\hat{\mathcal{L}}_{V}^{t}\right)^{*}$.

For $t$ fixed consider the transformation in the set of measures $\mu$ on $\Omega$ given by $\left(\hat{\mathcal{L}}_{V}^{t}\right)^{*}(\mu)=\nu$.

Theorem 1. For each $t$ there exists a probability measure $\nu_{V}$ on $(\Omega, \mathcal{B})$ which is fixed for such transformation $\left(\hat{\mathcal{L}}_{V}^{t}\right)^{*}$. The probability $\nu_{V}$ does not depend on $t$.

\section{Proof:}

We have to show that there exists $\nu_{V}$ such that for all $t>0$ and for all $g$ we have

$$
\int \hat{\mathcal{L}}_{V}^{t}(g) d \nu_{V}=\int g d \nu_{V}
$$


Remember that, $\hat{\mathcal{L}}_{V}^{t}(1)=1$, therefore, if $\mu$ is a probability, then $\left(\hat{\mathcal{L}}_{V}^{t}\right)^{*}(\mu)=$ $\nu$ is also a probability.

Denote by $\nu=\nu_{V}$ the probability obtained in the following way, for

$$
g=I_{\left\{X_{0}=a_{0}, X_{t_{1}}=a_{1}, X_{t_{2}}=a_{2}, \ldots, X_{t_{r-1}}=a_{r-1}, X_{r}=a_{r}\right\}},
$$

with $0<t_{1}<t_{2}<\ldots<t_{s-1}<t_{s}<. .<t_{r}$, we define

$$
\int g(w) d \nu(w)=e_{a_{r} a_{r-1}}^{\left(t_{r}-t_{r-1}\right)(L+V-\lambda I)} \ldots e_{a_{2} a_{1}}^{\left(t_{2}-t_{1}\right)(L+V-\lambda I)} e_{a_{1} a_{0}}^{t_{1}(L+V-\lambda I)} \mu_{V}\left(a_{0}\right) .
$$

It is easy to see that these probability transitions satisfy the Kolmogorov compatibility conditions. In section 4.1 and in Theorem 1.1 (same section) in $[\mathrm{EK}]$ it is described these conditions. We point out that the space we consider is separable and complete according to chapter 3 in the same book.

In order to show that $\nu$ is a probability we have to use the fact that $\sum_{c \in S} \mu_{V}(c)=1$

For example, $\int I_{\left\{X_{0}=c\right\}} d \nu=\mu_{V}(c)$. Moreover,

$$
\begin{gathered}
\int 1 d \nu=\sum_{c} \sum_{a} \int I_{\left\{X_{t}=c, X_{0}=a\right\}} d \nu=\sum_{c} \sum_{a} e_{c a}^{t(L-V-\lambda I)} \mu_{V}(a)= \\
\sum_{c} \mu_{V}(c)=1 .
\end{gathered}
$$

Suppose $t$ is such that $0<t_{1}<t_{2}<\ldots<t_{s-1}<t \leq t_{s}<. .<t_{r}$, then

$$
\begin{gathered}
z(w)=\hat{\mathcal{L}}_{V}^{t}(g)(w)= \\
\frac{1}{\mu_{V}(a)} e_{a a_{s-1}}^{\left(t-t_{s-1}\right)(L+V-\lambda I)} \ldots e_{a_{2} a_{1}}^{\left(t_{2}-t_{1}\right)(L+V-\lambda I)} e_{a_{1} a_{0}}^{t_{1}(L+V-\lambda I)} \mu_{V}\left(a_{0}\right),
\end{gathered}
$$

for $w$ such that $w(0)=a, w_{t_{s}-t}=a_{s}, w_{t_{s+1}-t}=a_{s+1}, \ldots, w_{t_{r}-t}=a_{r}$, and $\hat{\mathcal{L}}_{V}^{t}(g)(w)=0$ otherwise. Note that $z(w)=\hat{\mathcal{L}}_{V}^{t}(g)(w)$ depends only on $w_{0}, w_{t_{s}-t}, w_{t_{s+1}-t}, \ldots, w_{t_{r}-t}$.

We have to show that for any $g$ we have $\int g d \nu=\int \hat{\mathcal{L}}_{V}^{t}(g) d \nu$.

Now,

$$
\begin{gathered}
\int z(w) d \nu(w)=\int \sum_{c \in S} I_{\left\{X_{0}=c, X_{t_{s}-t}=a_{s}, X_{t_{s+1}-t}=a_{s+1}, \ldots, X_{t_{r}-t}=a_{r}\right\}} z(w) d \nu(w)= \\
\sum_{c \in S} \nu\left(\left\{X_{0}=c, X_{t_{s}-t}=a_{s}, X_{t_{s+1}-t}=a_{s+1}, \ldots, X_{t_{r}-t}=a_{r}\right\}\right) \\
\frac{1}{\mu_{V}(c)} e_{c a_{s-1}\left(t-t_{s-1}\right)(L+V-\lambda I)} \ldots e_{a_{2} a_{1}}^{\left(t_{2}-t_{1}\right)(L+V-\lambda I)} e_{a_{1} a_{0}}^{t_{1}(L+V-\lambda I)} \mu_{V}\left(a_{0}\right)=
\end{gathered}
$$

São Paulo J.Math.Sci. 4, 1 (2010), 1-16 


$$
\begin{aligned}
& \sum_{c \in S} e_{a_{r} a_{r-1}}^{\left(t_{r}-t_{r-1}\right)(L+V-\lambda I)} \ldots e_{a_{s+1} a_{s}}^{\left(t_{s+1}-t_{s}\right)(L+V-\lambda I)} e_{a_{s} c}^{t_{s}-t(L+V-\lambda I)} \mu_{V}(c) \\
& \frac{1}{\mu_{V}(c)} e_{c a_{s-1}}^{\left(t-t_{s-1}\right)(L+V-\lambda I)} \ldots e_{a_{2} a_{1}}^{\left(t_{2}-t_{1}\right)(L+V-\lambda I)} e_{a_{1} a_{0}}^{t_{1}(L+V-\lambda I)} \mu_{v}\left(a_{0}\right)= \\
& e_{a_{r} a_{r-1}}^{\left(t_{r}-t_{r-1}\right)(L+V-\lambda I)} \ldots e_{a_{s+1} a_{s}}^{\left(t_{s+1}-t_{s}\right)(L+V-\lambda I)} \\
& \left(\sum_{c \in S} e_{a_{s} c}^{\left(t_{s}-t\right)(L+V-\lambda I)} e_{c a_{s-1}}^{\left(t-t_{s-1}\right)(L+V-\lambda I)}\right) \ldots e_{a_{2} a_{1}}^{\left(t_{2}-t_{1}\right)(L+V-\lambda I)} e_{a_{1} a_{0}}^{t_{1}(L+V-\lambda I)} \mu_{V}\left(a_{0}\right)= \\
& , e_{a_{r} a_{r-1}}^{\left(t_{r}-t_{r-1}\right)(L+V-\lambda I)} \ldots e_{a_{s+1} a_{s}}^{\left(t_{s+1}-t_{s}\right)(L+V-\lambda I)} \\
& e_{a_{s} a_{s-1}}^{\left(t_{s}-t_{s-1}\right)(L+V-\lambda I)} \ldots e_{a_{2} a_{1}}^{\left(t_{2}-t_{1}\right)(L+V-\lambda I)} e_{a_{1} a_{0}}^{t_{1}(L+V-\lambda I)} \mu\left(a_{0}\right)= \\
& \int g d \nu
\end{aligned}
$$

The claim for the general $g$ follows from the above result.

Therefore, $\left(\hat{\mathcal{L}}_{V}^{t}\right)^{*}\left(\nu_{V}\right)=\nu_{V}$.

Definition 3.5. Consider the stationary probability $\rho_{V}=f_{V} \nu_{V}$ on $\Omega$. We call it the equilibrium state for $V$.

Definition 3.6. We call the probability $\nu_{V}$ on $\Omega$ the Gibbs state for $V$.

Proposition 3.7. For any integrable $f, g \in \mathcal{L}^{\infty}(P)$ and any positive $t$

$$
\int \hat{\mathcal{L}}_{V}^{t}(f) g d \nu_{V}=\int \hat{\mathcal{L}}_{V}^{t}\left(f\left(g \circ \theta_{t}\right)\right) d \nu_{V}=\int f\left(g \circ \theta_{t}\right) d \nu_{V} .
$$

Now we can prove our main result.

Theorem A. For any integrable $g \in \mathcal{L}^{\infty}(P)$ and any positive $t$

$$
\int e^{-\int_{0}^{t}\left(V \circ \Theta_{s}\right)(.) d s}\left[\left(\frac{1}{f_{V}} \mathcal{L}^{t}\left(e^{\int_{0}^{t}\left(V \circ \Theta_{s}\right)(.) d s} g f_{V}\right)\right) \circ \theta_{t}\right] d \nu_{V}=\int g d \nu_{V} .
$$

\section{Proof:}

Note first that if $w$ is such that $w(0)=c$, then

$$
\begin{gathered}
\mathcal{L}^{t}\left(f_{V}(w)\right)=\sum_{j} \mathcal{L}^{t}\left(I_{\left\{X_{0}=j\right\}}\right) f_{V}(j)=\sum_{j} \mathcal{L}^{t}\left(I_{\left\{X_{0}=j\right\}}\right) \frac{\mu_{V}(j)}{p_{j}^{0}}= \\
\sum_{j} \frac{1}{p_{c}^{0}} P_{c j} p_{j}^{0} \frac{\mu_{V}(j)}{p_{j}^{0}}=\sum_{j} \frac{1}{p_{c}^{0}} P_{c j} \mu_{V}(j)=\frac{\mu_{V}(c)}{p_{c}^{0}}=f_{V}(w) .
\end{gathered}
$$


Therefore, for all $w$

$$
\frac{1}{f_{V}} \mathcal{L}^{t}\left(f_{V}(w)\right)=1
$$

Finally,

$$
\begin{gathered}
\int e^{-\int_{0}^{t}\left(V \circ \Theta_{s}\right)(.) d s}\left[\left(\frac{1}{f_{V}} \mathcal{L}^{t}\left(e^{\int_{0}^{t}\left(V \circ \Theta_{s}\right)(.) d s} g f_{V}\right)\right) \circ \theta_{t}\right] d \nu_{V}= \\
\int e^{-\int_{0}^{t}\left(V \circ \Theta_{s}-\lambda\right)(.) d s}\left[\left(\frac{1}{f_{V}} \mathcal{L}^{t}\left(e^{\int_{0}^{t}\left(V \circ \Theta_{s}-\lambda\right)(.) d s} g f_{V}\right)\right) \circ \theta_{t}\right] d \nu_{V}= \\
\int\left[\hat{\mathcal{L}}_{V}^{t}\left(e^{-\int_{0}^{t}\left(V \circ \Theta_{s}-\lambda\right)(.) d s}\right)\right]\left[\frac{1}{f_{V}} \mathcal{L}^{t}\left(e^{\int_{0}^{t}\left(V \circ \Theta_{s}-\lambda\right)(.) d s} g f_{V}\right)\right] d \nu_{V}= \\
\int\left[\frac{1}{f_{V}} \mathcal{L}^{t}\left(e^{-\int_{0}^{t}\left(V \circ \Theta_{s}\right)(.) d s} e^{\int_{0}^{t}\left(V \circ \Theta_{s}\right)(.) d s} f_{V}\right)\right] \\
{\left[\frac{1}{f_{V}} \mathcal{L}^{t}\left(e^{\int_{0}^{t}\left(V \circ \Theta_{s}-\lambda\right)(.) d s} f_{V} g\right)\right] d \nu_{V}=} \\
\int\left[\frac{1}{f_{V}} \mathcal{L}^{t}\left(f_{V}\right)\right]\left[\frac{1}{f_{V}} \mathcal{L}^{t}\left(e^{\int_{0}^{t}\left(V \circ \Theta_{s}-\lambda\right)(.) d s} f_{V} g\right)\right] d \nu_{V}= \\
\int \frac{1}{f_{V}} \mathcal{L}^{t}\left(e^{\int_{0}^{t}\left(V \circ \Theta_{s}-\lambda\right)(.) d s} f_{V} g\right) d \nu_{V}=\int \hat{\mathcal{L}}_{V}^{t}(g) d \nu_{V}=\int g d \nu_{V} .
\end{gathered}
$$

\section{Referências}

[AGS] L. Ambrosio, N. Gigli, G. Savare, Gradient Flows in Metric Spaces and in the Space of Probability Measures., ETH Zurich, Birkhauser Verlag, Basel, (2005).

[B] P. Billingsley, Convergence of probability measures, John Wiley, (1968).

[BW] R. Bhattacharya and E. Waymire, Stochastic Processes with applications, Wiley (1990).

[CL] G. Castro and A. O. Lopes, "KMS States, Entropy and a Variational Principle for Pressure", Real Analysis Exchange, Vol 34, 2 (2009).

[EK] S. Ethier and T. Kurtz, Markov Processes, John Wiley, (1986).

[EL1] R. Exel and A. O. Lopes, " $C^{*}$-Algebras, approximately proper equivalence relations, and Thermodynamic Formalism", Erg. Theo. and Dyn. Systems. 24, 1051-1082, (2004).

[EL2] R. Exel and A. O. Lopes, "C*-Algebras and Thermodynamic Formalism", São Paulo Journal of Mathematical Sciences. 2, 1, 285-307, (2008).

[K] M. Kac, Integration in Function spaces and some of its applications, Acad Naz dei Lincei Scuola Superiore Normale Superiore, Piza, Italy, (1980).

[N] J. B. Norris, Markov Chains, Cambridge Press, (1998).

[P] K. Parthasarathy, Probability measures on metric spaces, AMS Chelsea, (2005).

[S] D. W. Strook, An introduction to Large Deviations, Springer, (1984). 